\title{
FRACTIONAL INTEGRAL ASSOCIATED WITH SCHRÖDINGER OPERATOR ON VANISHING GENERALIZED MORREY SPACES
}

\author{
Ali Akbulut, Ramin V. Guliyev, Suleyman Celik \\ AND MEHRIBAN N. OMAROVA
}

Abstract. Let $L=-\triangle+V$ be a Schrödinger operator, where the non-negative potential $V$ belongs to the reverse Hölder class $R H_{n / 2}$, let $b$ belong to a new $B M O_{\theta}(\rho)$ space, and let $\mathscr{I}_{\beta}^{L}$ be the fractional integral operator associated with $L$. In this paper, we study the boundedness of the operator $\mathscr{I}_{\beta}^{L}$ and its commutators $\left[b, \mathscr{I}_{\beta}^{L}\right]$ with $b \in B M O_{\theta}(\rho)$ on generalized Morrey spaces associated with Schrödinger operator $M_{p, \varphi}^{\alpha, V}$ and vanishing generalized Morrey spaces associated with Schrödinger operator $V M_{p, \varphi}^{\alpha, V}$. We find the sufficient conditions on the pair $\left(\varphi_{1}, \varphi_{2}\right)$ which ensures the boundedness of the operator $\mathscr{I}_{\beta}^{L}$ from $M_{p, \varphi_{1}}^{\alpha, V}$ to $M_{q, \varphi_{2}}^{\alpha, V}$ and from $V M_{p, \varphi_{1}}^{\alpha, V}$ to $V M_{q, \varphi_{2}}^{\alpha, V}, 1 / p-1 / q=\beta / n$. When $b$ belongs to $B M O_{\theta}(\rho)$ and $\left(\varphi_{1}, \varphi_{2}\right)$ satisfies some conditions, we also show that the commutator operator $\left[b, \mathscr{I}_{\beta}^{L}\right]$ is bounded from $M_{p, \varphi_{1}}^{\alpha, V}$ to $M_{q, \varphi_{2}}^{\alpha, V}$ and from $V M_{p, \varphi_{1}}^{\alpha, V}$ to $V M_{q, \varphi_{2}}^{\alpha, V}, 1 / p-1 / q=\beta / n$.

Mathematics subject classification (2010): 42B35, 35J10, 47H50.

Keywords and phrases: Fractional integral associated with Schrödinger operator, commutator, BMO, vanishing generalized Morrey space associated with Schrödinger operator.

\section{REFERENCES}

[1] A. Akbulut, O. KuZu, Marcinkiewicz integrals with rough kernel associated with Schrödinger operator on vanishing generalized Morrey spaces, Azerb. J. Math. 4 (1) (2014), 40-54.

[2] A. Akbulut, A. Eroglu, A. M. Najafov, Some embedding theorems on the Nikolskii-Morrey type spaces, Advances in Analysis, 2016, 1 (1), 18-26.

[3] A. Akbulut, V. S. Guliyev, M. N. Omarova, Marcinkiewicz integrals associated with Schrödinger operators and their commutators on vanishing generalized Morrey spaces, Bound. Value Probl. (2017) 2017:121.

[4] B. Bongionnin, E. Harboure, O. Salinas, Commutators of Riesz transforms related to Schödinger operators, J. Fourier Anal. Appl. 17 (1) (2011), 115-134.

[5] T. BUI, Weighted estimates for commutators of some singular integrals related to Schrödinger operators, Bull. Sci. Math. 138 (2) (2014), 270-292.

[6] X. CAO, D. CHEN, The boundedness of Toeplitz-type operators on vanishing-Morrey spaces, Anal. Theory Appl. 27 (4) (2011), 309-319.

[7] F. Chiarenza, M. Frasca, Morrey spaces and Hardy-Littlewood maximal function, Rend Mat. 7 (1987), 273-279.

[8] G. Di FAZIO, M. A. Ragus A, Interior estimates in Morrey spaces for strong solutions to nondivergence form equations with discontinuous coefficients, J. Funct. Anal. 112 (1993) 241-256.

[9] D. FAN, S. LU, D. YANG, Boundedness of operators in Morrey spaces on homogeneous spaces and its applications, Acta Math. Sinica (N. S.) 14 (1998), 625-634.

[10] V. S. GuliyeV, Boundedness of the maximal, potential and singular operators in the generalized Morrey spaces, J. Inequal. Appl. 2009, Art. ID 503948, 20 pp. 
[11] V. S. Guliyev, S. S. Aliyev, T. Karaman, P. Shukurov, Boundedness of sublinear operators and commutators on generalized Morrey spaces, Integral Equations and Operator Theory 71 (3) 2011, 327-355.

[12] V. S. GuLIYEV, Function spaces and integral operators associated with Schrödinger operators: an overview, Proc. Inst. Math. Mech. Natl. Acad. Sci. Azerb. 40 (2014), 178-202.

[13] C. Morrey, On the solutions of quasi-linear elliptic partial differential equations, Trans. Amer. Math. Soc. 43 (1938), 126-166.

[14] T. Mizuhara, Boundedness of some classical operators on generalized Morrey spaces, Harmonic Analysis (S. Igari, Ed.), ICM 90 Satellite Proceedings, Springer-Verlag, Tokyo (1991), 183-189.

[15] E. NAKAI, Hardy-Littlewood maximal operator, singular integral operators and the Riesz potentials on generalized Morrey spaces, Math. Nachr. 166 (1994), 95-103.

[16] M. A. RAGUSA, Commutators of fractional integral operators on vanishing-Morrey spaces, J. Global Optim. 40 (1-3) (2008), 361-368.

[17] N. S Aмко, Maximal, potential and singular operators in vanishing generalized Morrey spaces, J. Global Optim. 57 (4) (2013), 1385-1399.

[18] Z. SHEN, $L_{p}$ estimates for Schrödinger operators with certain potentials, Ann. Inst. Fourier (Grenoble) 45 (2) (1995), 513-546.

[19] L. Softova, Singular integrals and commutators in generalized Morrey spaces, Acta Math. Sin. (Engl. Ser.) 22 (3) (2006), 757-766.

[20] E. M. Stein, Harmonic Analysis: Real-variable Methods, Orthogonality, and Oscillatory Integrals, Princeton Univ. Press, Princeton, NJ, 1993.

[21] L. TANG, J. Dong, Boundedness for some Schrödinger type operator on Morrey spaces related to certain nonnegative potentials, J. Math. Anal. Appl. 355 (2009), 101-109.

[22] C. VitanZa, Functions with vanishing Morrey norm and elliptic partial differential equations, In: Proceedings of methods of real analysis and partial differential equations, Capri, pp. 147-150, Springer, 1990.

[23] R. Wheeden, A. Zygmund, Measure and integral, An introduction to real analysis, Pure and Applied Mathematics, 43, Marcel Dekker, Inc., New York-Basel, 1977. 